\title{
Аспекты оптимизации транспортировки экспортной продукции лесопромышленных предприятий
}

\section{Родион Рогулин}

Дальневосточный федеральный университет, г. Владивосток, Россия

\section{Информация о статье}

Поступила в редакциню:

12.08.2020

Принята

к опубликованию:

05.11 .2020

УДК 656.027

JEL L91

\begin{abstract}
Аннотация
Целью данной статьи является совершенствование методики оценки оптимальной степени заполняемости круглым лесом контейнеров для задачи транспортировки грузов через границь с другими странами. Задачами исследования является построение математической модели по оченке оптимальной степени загрузки контейнеров кругльм лесом и апробаиия ее на данных одного из лесопромышленных комплексов Российской Федерации. Обращено внимание на то, что в процессе транспортировки лесной продукции внешним потребителям время досмотра груза на таможне и риск нарушения сроков поставки могут существенно уменьшиться, если в контейнерах остается свободное пространство, что позволит провести таможенный досмотр с частичной выгрузкой товара. В работе представлены два подхода к моделированию прочессов транспортировки контейнеров с круглым лесом, реализующие численные типь расчетов и основанные на применении приемов имитационного моделирования. Рассмотрень наиболее часто встречаемье в науке методы и алгоритмы, моделируюшие любой сложности транспортные процессы. Представлено решение задачи оптимальной загрузки контейнеров при прохождении таможенного контроля. Реализована имитачионная модель транспортировки круглого леса через границы, которая позволила определить оптимальный объем загрузки контейнеров с целью минимизации временных затрат, необходимых для прохождения таможенного досмотра.
\end{abstract}

\section{Aspects of Optimizing the Transportation of Timber Industry Export Products}

Rodion Rogulin

\section{Abstract}

The purpose of this paper is to improve the methodology for assessing the optimal degree of the containers occupancy with the round timber for cargo transporting across the borders with other countries. The objectives of the research were to build a mathematical model to estimate the optimal degree of containers loading with the round timber and to test it on the data from one of the timber industry complexes of the Russian Federation. The attention was drawn to the fact that in the process of transporting timber products to external consumers, the time of cargo examination at the Customs and the risk of violating the delivery terms 
may be significantly reduced if there is a free space in the containers, which will allow the Customs examination to be made with partial unloading of goods. The paper presents two approaches to modeling the processes of transporting containers with round timber, implementing numerical types of calculations and based on the application of simulation modeling techniques. The methods and algorithms most frequently encountered in science that simulate transport processes of any complexity were considered. The solution to the issue of optimal containers loading during the Customs control was presented. A simulation model of round timber transporting across the borders was implemented, which allowed determining the optimal volume of container loading to minimize the time required for the Customs examination.

\section{Введение}

Процедура таможенного досмотра часто является фактором, влияющим на задержку сроков поставки товаров. Задержка поставки нередко приводит к отказу потребителя от заказанной продукции, и, как следствие, к потере прибыли и привлекательности предприятия на рынке сбыта. Пытаясь найти дополнительные пути оптимизации времени доставки конечных видов товаров до потребителя, компании стали заполнять по максимуму контейнеры с грузами, однако, это влечет лишь долгий процесс досмотра товаров.

Как известно, при таможенном досмотре экспортной лесопродукции используют полную или частичную выгрузку для фотосъемки бирок с каждой стороны круглой древесины. При этом помимо потери времени на досмотр происходит также незначительная деформация круглого леса, что отрицательно сказывается на отношениях между покупателем и продавцом.

Проблемы транспортировки экспортной продукции рассмотрены в ряде научных работ. В работе [2] описаны основные проблемы нормативного документального обеспечения трансграничных железнодорожных перевозок, связанные с вопросами пересечения границы и маневрирования на станциях на примере Польши и двух соседних стран (Германии и Чехии). Основной вывод авторов [2] заключается в необходимости рассмотрения возможности обновления местных пограничных соглашений с целью сокращения объема этих документов, унификации, упрощения и удаления ненужных записей.

Существует мнение, что для решения проблемы межгосударственных перевозок необходимы соответствующие экономические коридоры, которые снизят затраты производителей-экспортеров и увеличат их прибыль. Этот вариант рассматривался в статье [4] на примере экономики Индии. Одним из важных выводов, сделанных в отношении политики в отношении транспортных коридоров, является риск того, что будущий рост останется сконцентрированным в районах, непосредственно связанных с предлагаемым коридором, в результате чего периферийные районы будут маргинализованы. В целях предотвращения или даже уменьшения диспропорций авторы [4] рекомендуют разработать пространственные стратегии с механизмами, позволяющими их реализовать на более низких уровнях государственного управления, реформировать территориальные планы и расходы на инфраструктуру для улучшения доступности между предлагаемыми коридорами и периферийными районами и реализацию политики развития транспорта.

Исходя из обозначенных проблем транспортировки экспортной продукции, применительно к специфике лесопромышленных предприятий,-целью данной работы является совершенствование методики оценки оптимальной заполняемости круглым лесом транспортных контейнеров. Задачами исследования являются, во-первых, составление математической модели по имитации процесса прохождения груза с фиксированным значением наполненности контейнера через границы с государствами, во-вторых, расчет оптимального значения загрузки контейнера. Гипотеза данного исследования - предположение о том, что значение оптимального заполнения лесом контейнера лежит в пределах 60-70\%. 
В работе использованы данные предприятия центральной части России, экспортирующего лесопродукцию в Германию и Польшу через Украину и Чехию.

\section{Постановка проблемы}

Любое производственное предприятие стремится увеличить объем производства и расширить рынки сбыта произведенной продукции за счет совершенствования технологических процессов, что позволит получить дополнительную прибыль [5, 6, 7]. На лесопромышленных предприятиях основным производственным процессом является получение товарной продукции из различных видов древесного сырья. Укрупненная схема основных производственных процессов предприятия лесной промышленности представлена на рис. 1.

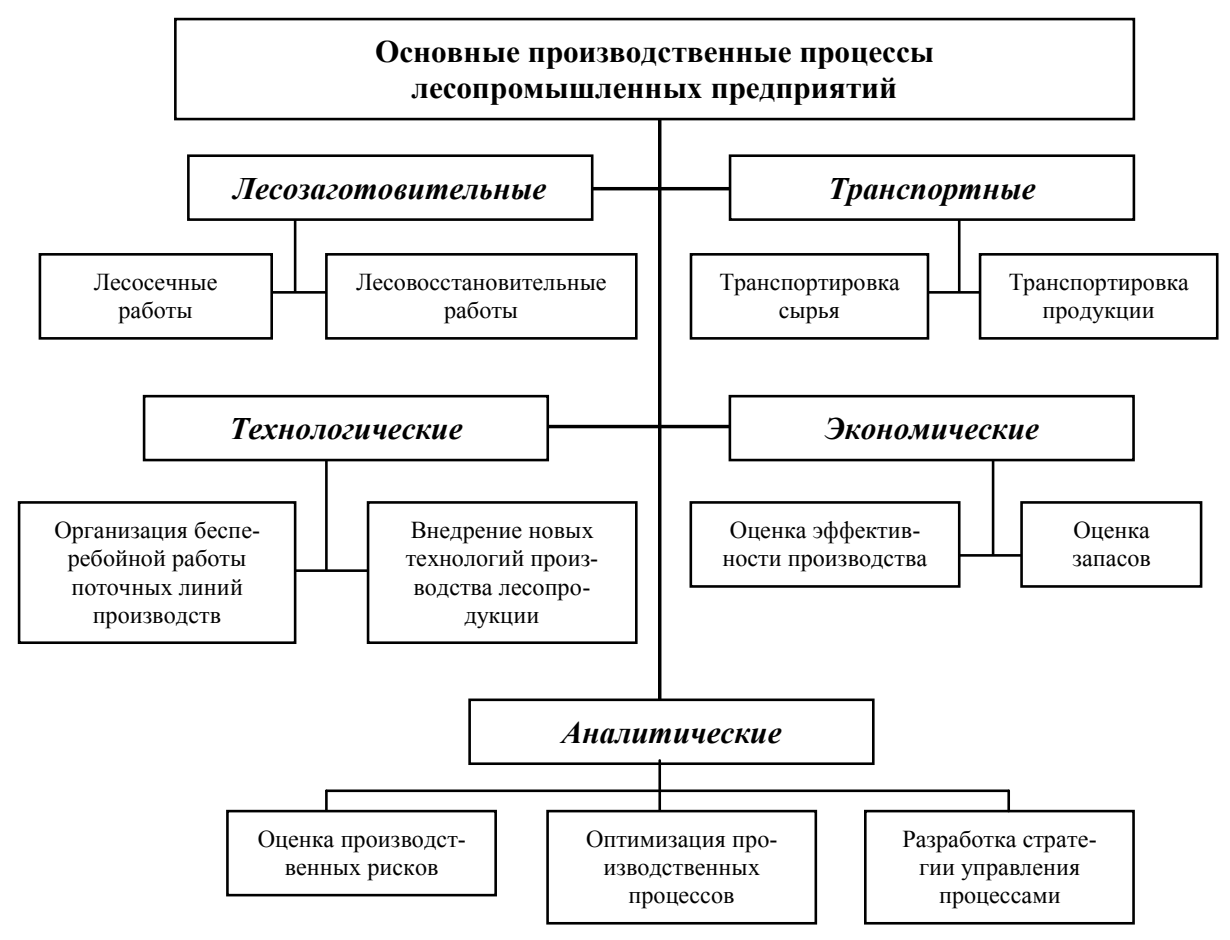

Puc. 1. Укрупненная схема основных производственных процессов предприятия лесной промышленности

Источник: составлено автором

Транспортировка сырья и готовой продукции является одним из важнейших производственных процессов лесопромышленного предприятия, который связывает все этапы производства и реализации лесопродукции (рис. 2).

Процесс транспортировки сопровождается значительными затратами времени и финансовых ресурсов. Грузы для потребителя могут быть отправлены как непосредственно с лесосеки, так и с транзитно-перевалочного терминала или склада готовой продукции $[8,9]$. Но все грузы, предназначенные для внешних потребителей, проходят процедуру таможенного досмотра на контрольнопропускных пунктах (далее - КПП). Потери времени при прохождении границы считаются простоем и увеличивает затраты, связанные с международными перевозками лесопродукции. Таможенный досмотр - один из факторов, который значительно замедляет процесс транспортировки продукции, что может привести к нарушению сроков поставки продукции и в итоге к потере прибыли $[10,11$, 
12]. Как правило, досмотр груза на таможенных пунктах проходит путем полной выгрузки содержимого контейнера и последующим осмотром каждой отдельной единицы товара, и сверкой маркировок на древесине с номерами, занесенными Единую Государственную Автоматизированную Информационную Систему (ЕГАИС). Однако, время досмотра груза может существенно уменьшиться, если в контейнерах остается свободное пространство, что позволит провести таможенный досмотр с частичной выгрузкой товара. Этот прием может позволить существенно сократить время досмотра контейнера. Соотношение свободного и занятого пространства при загрузке контейнера ${ }^{1}$ не является очевидным, и мы в своей статье хотим показать, что неполное заполнение контейнера грузом может привести к значительному экономическому эффекту $[13,14,15]$.

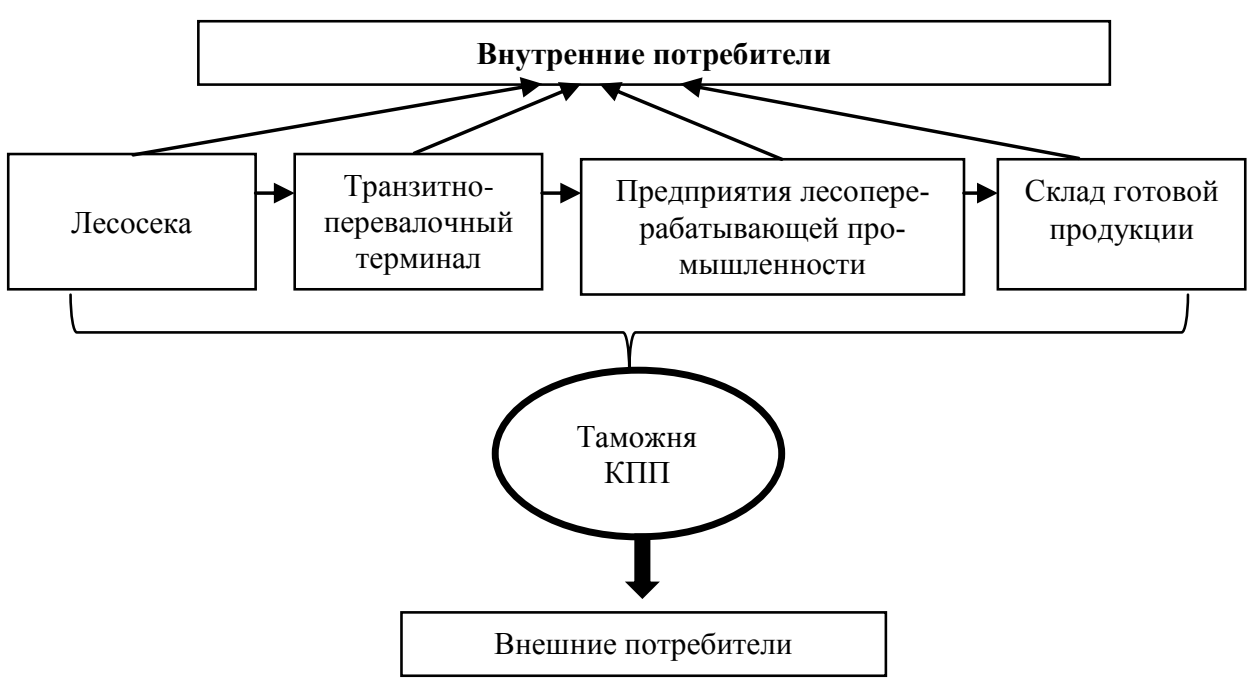

Puc. 2. Схема транспортных потоков на предприятиях лесной промышленности Источник: составлено автором

Представим модель исследования в общем виде. Пусть существует $n$ заводов (пунктов отправки), $m$ заказчиков (покупателей, потребителей), $u$ границ, которые предстоит пересечь товару, $T$ - это время для таможенного досмотра продукции. Пусть транспортная система такая, что, во-первых, $t_{i}$ - временные затраты на доставку по $i$-ой дороге, во-вторых, $s_{i}$ - расстояние на доставку по $i$-ой дороге, с - затраты бензина на 1 км пути, $t_{v}$ максимальное время ожидания $v$-ым покупателем груза, $b$ - это то время, сверх которого покупатель ждет купленного товара, вероятность $p_{1 j}$ контроля сотрудников пограничного контроля на $j$ посту, вероятность $p_{2 v}$ отказа $v$-го заказчика от покупки ввиду дополнительного времени ожидания, $U$ - количество загруженных автомобилей, $k$ - процент загрузки автомобиля, $p$-цена реализации товара. Будем считать, что автомобили принадлежат компании-отправителю, которая не несет издержки на их аренду и амортизацию. Транспорт будет выбирать наикратчайший путь до своей цели.

Введем понятие выручки $\left(R_{i}\right)$ при успешной доставке $i$ товара и форму расчета:

$$
R_{i}=\left\{\begin{array}{c}
p, \text { если товар доставлен до потребителя } \\
0, \text { иначе. }
\end{array}\right.
$$

\footnotetext{
${ }^{1}$ В основном на практике встречается 98-100\% загрузка контейнеров, однако, в данной работы мы усомнимся в том, что это оптимальная степень заполняемости.
} 
Введем понятие денежных издержек $\mathrm{C}_{i}$ при транспортировке груза по трассе $i$ :

$$
C_{i}=c * s_{i} .
$$

Введем понятие прибыли, как:

$$
\pi=T R-T C=\sum R_{i}-\sum C_{i}
$$

где $T R$ - суммарная выручка, $T C$ - суммарные издержки. Определим следующие параметры, как функции:

$$
\begin{aligned}
& p_{2 j}=f(b) \\
& p_{1 j}=f(k) \\
& U=g(k)
\end{aligned}
$$

При пересечении границы происходит расчет $p_{1 j}$. Далее алгоритм выдает, проверку или отсутствие проверки груза на границе. В случае проверки его на границе издержек на бензин нет, кроме издержек $T$. В случае отсутствия контроля на границе транспорт проезжает дальше, не теряя времени. Если в ходе транспортировки потребитель отказывается от покупки товара ${ }^{2}$ транспорт направляется на заведомо обговоренный склад и не пересекает дополнительно каких-либо границ, но несет также все транспортные издержки $c$. Выручка зачисляется на счет продавца в момент получения товара покупателем, после транспорт остается в том же пункте и не несет дополнительных издержек.

\section{Методы решения и алгоритмы}

Для решения подобной задачи необходимо рассмотреть два подхода. При первом подходе используются численные типы расчета. Примером реализации является аналитический метод на основе «генетического алгоритма». На первом этапе строится математическая непрерывная или дискретная модель. На втором - происходит «размножение», «мутация» решений. После анализа значений целевой функции при таких наборах решения определяется лучшая «особь» (процесс «селекции»), ее дальше планируется «размножать» $[12,13,14]$.

Второй подход представляет собой имитационное решение задачи оптимизации. В программе, реализующей имитационную модель, можно выделить аналоги устройствам и элементам реальной системы, условиям их функционирования. Программа имитации воспроизводит состав исследуемой системы, процессы функционирования системы во времени, при этом имитируются элементарные явления, составляющие процесс с сохранением их логической структуры и последовательности протекания во времени $[5,19,20,21]$. Основные алгоритмы, реализующие эти два подхода, представлены в табл. 1.

Для решения задачи оптимальной загрузки контейнеров был выбран алгоритм имитационного моделирования, так как он дает возможность учитывать фактор времени.

Этот выбор основан на том, что трудно провести статистическую оценку степени наполненности контейнеров из-за достаточно большого количества случайных переменных в то время, как имитационное моделирование - это полез-

\footnotetext{
${ }^{2}$ На случай форс-мажорной ситуации, когда круглый лес не поступает вовремя на производство происходит остановка производства, что влечет за собой соответствующие экономические потери. Кроме того, у леса есть очень ограниченный срок годности, что также может сказаться на качестве конечной продукции, если производить из такого материала товары. Поэтому предприятия отказываются от круглого леса, который поступает не вовремя.
} 
ный вид моделирования в ситуациях, когда имеет место большое количество стохастических параметров.

Таблицча 1

Основные алгоритмы решения задачи оптимальной загрузки

\begin{tabular}{|c|c|}
\hline Краткое описание & Особенности \\
\hline $\begin{array}{l}\text { Линейное стохастическое программирование } \\
{[22,23] \text { - подход в математическом программи- }} \\
\text { ровании, позволяющий учитывать неопределён- } \\
\text { ность в оптимизационных линейных моделях. }\end{array}$ & $\begin{array}{l}\text { В то время как детерминированные задачи оптимизации } \\
\text { формулируются с использованием заданных параметров, } \\
\text { реальные прикладные задачи обычно содержат некоторые } \\
\text { неизвестные параметры. Когда параметры известны толь- } \\
\text { ко в пределах определенных границ, один подход к реше- } \\
\text { нию таких проблем называется робастной оптимизацией. } \\
\text { Этот подход состоит в том, чтобы найти решение, кото- } \\
\text { рое является допустимым для всех таких данных и в не- } \\
\text { котором смысле оптимально. }\end{array}$ \\
\hline $\begin{array}{l}\text { Генетический алгоритм }[24,25] \text { представляет } \\
\text { собой эвристический метод случайного поиска, } \\
\text { основанный на принципе имитации эволюции } \\
\text { биологической популяции. Обновление попу- } \\
\text { ляции выполняется некоторое (заданное) число } \\
\text { раз, и результатом работы алгоритма является } \\
\text { лучшее из найденных покрытий. }\end{array}$ & $\begin{array}{l}\text { В общем случае в процессе работы алгоритма происходит } \\
\text { последовательная смена популяций, каждая из которых } \\
\text { является семейством покрытий, называемых особями по- } \\
\text { пуляции. Покрытия начальной популяции строятся случай- } \\
\text { ным образом. Наиболее распространённая и лучше всего } \\
\text { зарекомендовавшая себя - стационарная схема генетиче- } \\
\text { ского алгоритма, в которой очередная популяция отличает- } \\
\text { ся от предыдущей лишь одной или двумя новыми особями. }\end{array}$ \\
\hline $\begin{array}{l}\text { Агентное моделирование }[26,27]-\text { направление } \\
\text { в имитационном моделировании, которое ис- } \\
\text { пользуется для исследования децентрализован- } \\
\text { ных систем, динамика функционирования кото- } \\
\text { рых определяется не глобальными правилами и } \\
\text { законами (как в других парадигмах моделирова- } \\
\text { ния), а наоборот, когда эти глобальные правила и } \\
\text { законы являются результатом индивидуальной } \\
\text { активности членов группы. }\end{array}$ & $\begin{array}{l}\text { Цель агентных моделей - получить представление об } \\
\text { этих глобальных правилах, общем поведении системы, } \\
\text { исходя из предположений об индивидуальном, частном } \\
\text { поведении её отдельных активных объектов и взаимодей- } \\
\text { ствии этих объектов в системе. Агент - некая сущность, } \\
\text { обладающая активностью, автономным поведением, мо- } \\
\text { жет принимать решения в соответствии с некоторым } \\
\text { набором правил, взаимодействовать с окружением, а } \\
\text { также самостоятельно изменяться. }\end{array}$ \\
\hline
\end{tabular}

Источник: составлено автором на основе [22-27]

\section{Обсуждение результатов}

Пусть производители со своих заводов отправляют круглый лес автотранспортом через границы Российская Федерация - Беларусь (Украина) - Европейский Союз. В качестве функции распределения вероятностей $f(b), f(k)$, (формулы 4-5), возьмем сигмоиду (рис. 3). По горизонтальной оси отложены значения $b$ или $k$, по вертикальной оси вероятности $f(b)$ или $f(k)$ соответственно.

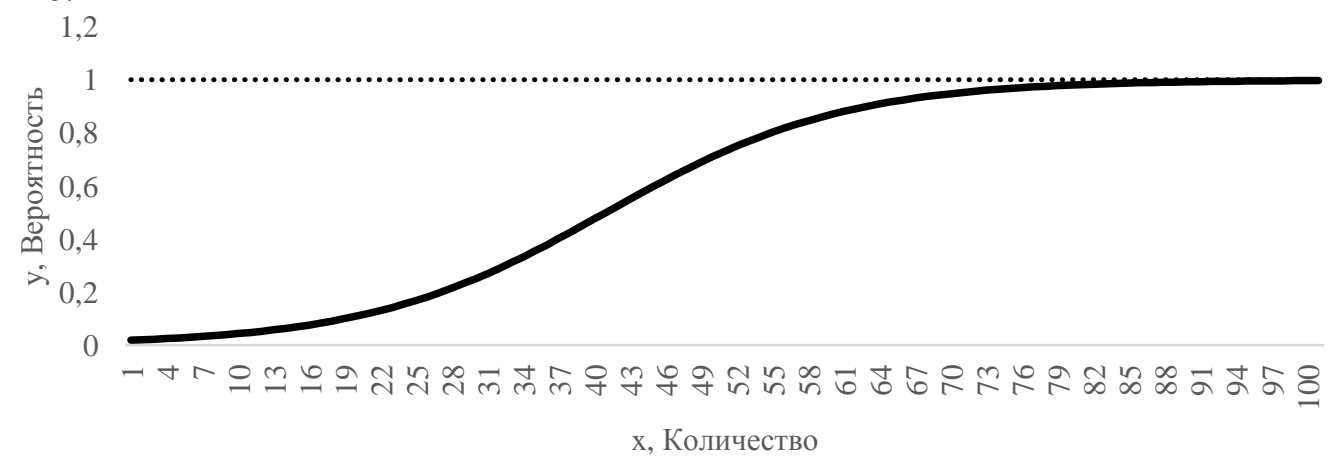

Сигмоида …...... Предел $\mathrm{f}(\mathrm{x})$

Рис. 3. Визуализация функции $f$

Источник: составлено автором

В случае отказа от товара автомобиль с грузом везет его до обусловленного пункта (склада), что тоже подразумевает экономические потери. 
Сам процесс был реализован средствами высокоуровнего языка программирования JavaScript (JS), программный код представлен в [28].

На первом шаге была загружена карта Центральной и Восточной Европы из соответствующего пакета (leaflet) прикладных программ языка программирования JS, включающая длины и пропускные способности дорог.

Затем был смоделирован процесс перевозки в контейнерах круглого леса длиною до 4 м и диаметром от 32 до 40 см одной из лесоперерабатывающей компании, расположенной на территории Российской Федерации. Количество автотранспорта не ограничено, расходы на заказ автотранспорта равны 0, считается, что автотранспорт принадлежит компании, но в случае использования каждой дополнительной единицы транспорта увеличатся расходы на бензин, таким образом представляется возможным учитывать количество используемого транспорта. Заметим, что верхней границей ограничения будет выступать расходы на бензин $c$, а нижнем ограничением будет выступать минимальное заполнение транспорта $(k)$. Алгоритм выбирает кратчайший путь. Места расположения производств: Московская и Псковская области, Волгоград. Склад расположен в г. Вроцлав. Потребители находятся в Гданьске, Берлине, Дрездене, Праге, Варшаве. Количество перевозимого товара будет в рассматриваемых ситуациях одним и тем же, но случайно генерируемый. Заказ может быть осуществлен по одному из двух тарифов: $p=100$ у. е. за 1 ед. товара и $p=200$ у. е. за 1 ед. товара, при этом заказ по большей стоимости будет исполнен в первую очередь. Стоимость бензина для перевозки на 1 км составляет $c=0,1 \mathrm{y}$. е.

В процессе имитационного моделирования эта задача перевозки лесопродукции была запущена 10000 раз, средние значения наиболее показательных результатов имитационного моделирования представлены в табл. 2. Показан процент от общего числа автомашин, не нарушивших сроки поставки по договору вследствие потерь времени при досмотре товаров на КПП таможни.

Таблица 2

Средние значения результатов имитационного моделирования

\begin{tabular}{|c|c|c|c|c|c|}
\hline $\begin{array}{l}\text { Загрузка кон- } \\
\text { тейнеров, \% }\end{array}$ & $\begin{array}{c}\text { А/м, не нару- } \\
\text { шившие сроки } \\
\text { поставки, \% }\end{array}$ & $\begin{array}{c}\text { Условная } \\
\text { прибыль, у. е. }\end{array}$ & $\begin{array}{c}\text { Количество } \\
\text { транспорта, } \\
\text { шт. }\end{array}$ & $\begin{array}{c}\text { Среднее число } \\
\text { a/м, не нару- } \\
\text { шивших срок } \\
\text { поставки, шт. }\end{array}$ & $\begin{array}{c}\text { Средняя приве- } \\
\text { денная при- } \\
\text { быль, у. е. }\end{array}$ \\
\hline 1 & 2 & 3 & 4 & 5 & 6 \\
\hline 10 & 39 & -108.33 & 1251 & 410 & -0.26 \\
\hline 20 & 38 & -16.58 & 660 & 216 & -0.08 \\
\hline 30 & 35 & 17.65 & 510 & 167 & 0.11 \\
\hline 40 & 33 & 23.85 & 392 & 128 & 0.19 \\
\hline 54 & 35 & 62.63 & 266 & 87 & 0.72 \\
\hline 56 & 32 & 64.02 & 260 & 85 & 0.75 \\
\hline 60 & 23 & 53.23 & 234 & 77 & 0.69 \\
\hline 62 & 34 & 59.65 & 229 & 75 & 0.79 \\
\hline 64 & 28 & 51.09 & 225 & 74 & 0.69 \\
\hline 66 & 35 & 57.97 & 220 & 72 & 0.80 \\
\hline 70 & 32 & 57.07 & 210 & 69 & 0.83 \\
\hline 74 & 30 & 28.95 & 201 & 66 & 0.44 \\
\hline 78 & 34 & 23.02 & 190 & 62 & 0.37 \\
\hline 80 & 38 & 56.96 & 188 & 62 & 0.92 \\
\hline 82 & 32 & 19.07 & 178 & 58 & 0.33 \\
\hline 84 & 31 & 28.29 & 170 & 56 & 0.51 \\
\hline 90 & 15 & -55.37 & 161 & 53 & -1.05 \\
\hline 92 & 14 & -63.54 & 160 & 52 & -1.22 \\
\hline 100 & 14 & -78.87 & 158 & 50 & -1.58 \\
\hline \multicolumn{3}{|c|}{$\begin{array}{c}\text { Средний процент a/м, } \\
\text { не нарушивших сроки поставки - } 33\end{array}$} & \multicolumn{3}{|c|}{ Максимальная прибыль - 0.92 у. е. } \\
\hline
\end{tabular}


Анализируя табл. 2, можно заметить, что на интервале степени заполнения контейнера 24-86\% прибыль положительна, максимальная условная прибыль, полученная в процессе имитационного моделирования, соответствует загрузке контейнера на 54-56\%. Однако при этом потребуется большее количество автомашин, с наполовину пустыми контейнерами. Рассчитаем приведенную прибыль - это средняя прибыль в расчете на одну машину, доехавшую с минимальным простоем на контрольно-пропускном пункте таможни и выдержавшая сроки договора поставки. Она будет максимальна при загрузке контейнера на $80 \%$, что опровергает искомую гипотезу. При такой загрузке не потребуется полностью освобождать контейнер для досмотра, что существенно снизит потери времени при пересечении границы.

На рис. 4 представлена визуализация параметра «приведенная прибыль» в зависимости от процента загрузки контейнера.

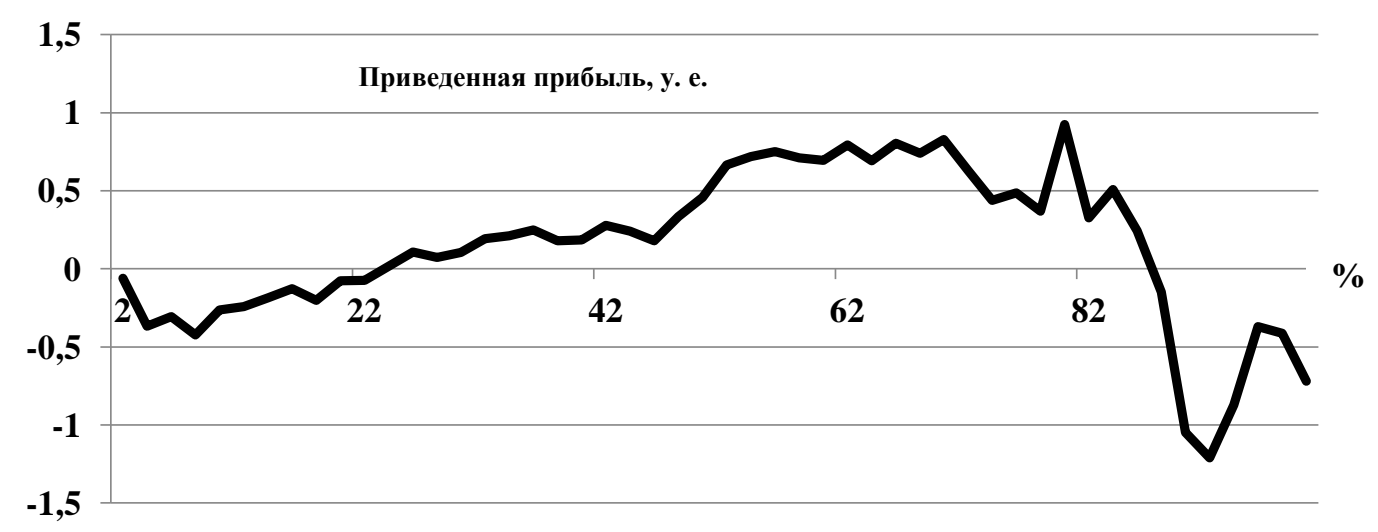

Puc. 4. Зависимость прибыли в пересчете на каждый автомобиль, доехавший с минимальным простоем на КПП таможни (у. е.) от степени заполнения контейнера (\%) ${ }^{3}$ Источник: составлено автором

Результаты имитационного моделирования позволяют сделать вывод, что заполнение на 100\% контейнеров, перевозящих лесопродукцию, ведет к временным потерям при пересечении границ и прохождении таможенного досмотра груза.

\section{Заключение}

В работе представлена экономико-математическая модель, позволяющая на теоретическом уровне вычислить оптимальное значение степени загрузки контейнеров круглым лесом для достижения максимального значения приведенной прибыли. Модель отличается возможностью учета вероятностных факторов досмотра груза на границах государств.

Международные перевозки лесопродукции и круглого леса автомобильным транспортом являются высокозатратным процессом, что объясняется транспортно-технологическими и организационными факторами [29]. Предложенный вариант неполной загрузки контейнера позволит сократить время таможенного досмотра, что существенно снижает затраты на транспортировку лесопродукции, а также снизит риск отказа клиента от продукции, доставленной с нарушением сроков поставки.

Показано, что загрузка на 100\% контейнера не дает желаемый эффект. Напротив, неполная загрузка контейнера на уровне $80 \%$ гарантирует оптимальное

\footnotetext{
${ }^{3}$ По оси Ох отложены значения степени наполненности контейнера.
} 
значение прибыли. К сожалению, начальная гипотеза о диапазонах степени загрузки контейнера не подтвердилась, но оказалась довольно близкой к оптимальному значению.

\section{Список источников / References}

1. Haradhan M. The First Industrial Revolution: Creation of a New Global Human Era // Journal of Social Sciences and Humanities, 2019, Vol. 5, p. 377-387.

2. Gamon, W., Naranjo Gómez, J.M. Main Problems of Railway Cross-Border Transport Between Poland, Germany and Czech Republic // Sustainability, 2019, Vol. 11, p. 1-10.

3. Kołodziejczyk Krz. Cross-border public transport between Poland and Czechia and the development of the tourism functions of the region // Geographia Polonica, 2020, Vol. 93, iss. 2, p. 261-285.

4. Jain M., Jehling M. Analysing transport corridor policies: An integrative approach to spatial and social disparities in India // Journal of Transport Geography, 2020, Vol. 86, p. 1-10. DOI:10.1016/j.jtrangeo.2020.102781

5. Рогулин Р.С., Мазелис Л.С. Алгоритм и математическая модель формирования устойчивых цепочек поставок древесного сырья из регионов России: сравнение и анализ // Вестник Пермского университета. Сер. «Экономика», 2020, № 3, с. 385-404. DOI: 10.17072/1994-9960-2020-3-385-404

6. Rogulin R.S. Going to Business or Intending in Science? // Экономика науки, 2020, Vol. 6, No. 3, pp. 169-179. DOI: https://doi.org/10.22394/2410-132X-2020-6-3-169-179

7. Рогулин Р. С. Построение нетривиальной экономико-математической модели для менеджмента производственных компаний // Российский экономический журнал, 2020, № 1, c. 108-116. DOI: 10.33983/0130-9757-2020-1-108-116

8. Безрукова Т.Л., Зиновьева И.С. Концептуальные положения обеспечения эффективного использования лесных ресурсов // Социально-экономические явления и процессы, 2017, т.12, №6, С.28-35

9. Олейник Е.Б. Анализ структурной динамики экспорта лесопродукции из Дальневосточного региона // Транспортное дело России, 2017, № 6 (ч. 1), С. 3-7.

10. Lim S. M., Sultan A. B. Md., Sulaiman Md. N., Mustapha A., Leong K. Y. Crossover and Mutation Operators of Genetic Algorithms // International Journal of Machine Learning and Computing, 2017, vol. 7, no. 1, pp. 9-12

11. Sumathi P. A new approach to solve linear programming problem with intercept values // Journal of Information and Optimization Sciences, 2016, vol. 37 (4), c.495510, DOI: $10.1080 / 02522667.2014 .996031$

12. Daganzo C. F., Smilowitz K. R. Bounds and approximations for the transportation problem of linear programming and other scalable network problems // Transportation Science, 2004, 38(3), p.343-356. DOI: 10.1287/trsc.1030.0037

13. Gharehbolagh H. H., Hafezalkotob A., Makui A., Raissi S. A cooperative game approach to uncertain decentralized logistic systems subject to network reliability considerations // Kybernetes, 2017, vol. 46, no. 8, pp. 1452-1468

14. Maysara S., Hendry L. C., Zorzini B. M. Institutional complexity and sustainable supply chain management practices // Supply Chain Management: An International Journal, 2017, Vol. 22 Issue: 6, pp.542-563, URL: https://doi.org/10.1108/ SCM-10-2016-0365

15. Рогулин Р.С., Нечаев П.В., Плешанов Д.Е., Олейник Е.Б. Комплексное решение задачи оптимизации процессов производства и транспортировки продукции // Вопросы экономики и права, 2018, №7(121), С.81-85

16. Dameshghi A., Hossein R. M. Wind turbine gearbox condition monitoring and fault diagnosis based on multi-sensor information fusion of SCADA and DSER-PSO-WRVM method // International Journal of Modelling and Simulation, 2019, 39:1, pp. 48-72, DOI: 10.1080/02286203.2018.1476008

17. Ushemadzoro Ch., Krenz P. M., Shawn C. From Antenna Design to High Fidelity, Full Physics Automotive Radar Sensor Corner Case Simulation // Modelling and Simulation in Engineering, 2018, Vol. 4, pp. 123-126. https://doi.org/10.1155/2018/4239725. 
18. Sonker Bh., Deepak K., Paulson S. Design of two degree of freedom-internal model control configuration for load frequency control using model approximation // International Journal of Modelling and Simulation, Vol. 39, pp. 27-37, DOI: 10.1080/02286203.2018.1474027

19. Zhang Y., Preetha Ph., Siyuan L., Ming Y., Bin L. Sh. W. Comparison of machine learning methods for stationary wavelet entropy-based multiple sclerosis detection: decision tree, $\mathrm{k}$ nearest neighbors, and support vector machine // Simulation, 2016, vol. 92(9), pp. 861-871. doi: 10.1177/0037549716666962

20. Leilei Zh., Yuewei Y., Changcheng Zh., Shaofang M., Fuxing Y. Simulation of vertical characteristics and in-wheel motor vibration of electric vehicles with asymmetric suspension damper under road impact // International Journal of Modelling and Simulation, 2019, Vol. 39:1, 14-20, DOI: 10.1080/02286203.2018.1468991

21. Olaru A., Olaru S., Mihai N. Modeling, Simulation and Assisted Research with LabVIEW Instrumentation in Robotic // International Journal of Modeling and Optimization, 2018, vol. 8, no. 6, pp. 301-305

22. Hosseinpour M., Hojjat Sh., Yasser Sh. Stepwise regression modeling for compressive strength assessment of mortar containing metakaolin // International Journal of Modelling and Simulation, 2018, Vol. 38:4, 207-215, DOI: 10.1080/02286203.2017.1422096

23. Jomsri P. Implementing Virtual 3D Model and Augmented Reality Navigation for Library in University // International Journal of Modeling and Optimization, 2018, vol. 8, no. 6, pp. 315-317

24. Chanda U., Kumar A., Kumar J. D. Fuzzy EOQ model of a high technology product under trial-repeat purchase demand criterion // International Journal of Modelling and Simulation, 2018, Vol. 38:3, pp. 168-179, DOI: 10.1080/02286203.2017.1407991

25. Bindu S., Thomas V. A. Modified Direct-Quadrature Axis Model for Characterization of Air-gap Mixed Eccentricity Faults in Three-Phase Induction Motor // International Review on Modelling and Simulations, 2018, vol. 11 (6), pp. 359-365. doi: https: //doi.org/10.15866/iremos.v11i6.15513

26. Attia H., Artificial Neural Networks 2018 Based Maximum Power Point Tracking Photovoltaic System for Remote Park LED Lighting Applications // International Review on Modelling and Simulations, 2018, Vol. 11 (6), pp. 396-405. doi: https: //doi.org/10.15866/iremos.v11i6.16165

27. Fakharzadeh J. A., Ebrahimi M. Z. A new outlier detection method for high dimensional fuzzy databases based on LOF // Journal of Mathematical Modeling, 2018, 6(2), pp. 123136. doi: $10.22124 / \mathrm{jmm} .2018 .8102 .1108$

28. Программный код, 2019, URL: https://github.com/FireEagle25/transport_article

29. Безрукова Т.Л., Животягина Н.И., Гвоздева Т.Е., Зазыкина Л.А. Проблемы и факторы, определяющие потребность лесного сектора экономики в государственной поддержке // Международный журнал прикладных и фундаментальных исследований, 2016, №4-2, C.432-435

\section{Сведения об авторе / About author}

Рогулин Родион Сергеевич, ассистент кафедры прикладной математики, механики, управления и программного обеспечения Школы естественных наук, Дальневосточный федеральный университет. Ассистент кафедры математики и моделирования, Владивостокский государственный университет экономики и сервиса. 690922 Россия, г. Владивосток, о-в Русский, кампус ДВФУ, корпус L. E-mail: rafassiaofusa@mail.ru

Rodion S. Rogulin, Assistant of the Department of Applied Mathematics, Mechanics, Control and Software, School of Natural Sciences, Far Eastern Federal University. Assistant of the Department of Mathematics and Modeling, Vladivostok State University of Economics and Service. Building L, FEFU campus, Russky Island, Vladivostok, Russia 690922. E-mail: rafassiaofusa@mail.ru 